MATEC Web of Conferences 22,01021 (2015)

DOI: $10.1051 /$ matec conf/20152201021

(C) Owned by the authors, published by EDP Sciences, 2015

\title{
Clustering Routing Algorithm in Wireless Sensor Networks Based on artificial Bee Colony and Assistant Cluster Heads
}

\author{
Yang Yang \& Guowei Fu \\ Department of Information Science and Electronic Engineering, Zhejiang University, Hangzhou, \\ Zhejiang, China
}

\begin{abstract}
The solution presented in this article is a new clustering routing algorithm based on artificial bee colony and assistant cluster-head. This algorithm is a selection process of optimizing master cluster-head and assistant cluster-head by introducing assistant cluster-head in the cluster and artificial bee colony algorithm. Simulation experiment shows that the clustering routing algorithm based on artificial bee colony and assistant cluster-head can effectively improve energy utilization ratio, balance network load and extend network lifetime.
\end{abstract}

Keyword: wireless sensor networks; route; artificial bee colony; assistant cluster-head; lifetime

\section{INTRODUCTION}

The wireless sensor networks [1] consisted of distributed sensor nodes have received broad attention in recent years, with extensive application background, such as military, environmental monitoring, medical attention [2] and etc., which transmit data to base station by cooperation method. Because node power is supplied by limited energy and irreplaceable battery, both energy utilization ratio and network energy consumption load balance are critical issues for wireless sensor networks. Research shows that wireless sensor networks adopting clustering routing protocol can extend network lifetime.

Protocol LEACH [3] is of self-organization and self-adaption clustering protocol, presenting that each node can be cluster-head based on a certain probability and cycle. Cluster header nodes receive data from other nodes inside the cluster, and reduce data redundancy by information fusion, then transmit these data to base station, whereas, in Protocol LEACH, cluster-head is selected randomly. Protocol ABC [4] is upgraded based on Protocol LEACH, the biggest difference between them is cluster-head selection is optimized by artificial bee colony algorithm, which can effectively improve energy utilization ratio, but cluster header nodes need to complete some works, such as data collection, information fusion, communication with base station, etc., also in case of heavy energy load that would lead cluster header nodes to die earlier.

The solution presented in this article is clustering routing algorithm based on artificial bee colony and assistant cluster-head (ABC-A), introducing assistant cluster-head mechanism and optimizing selection for cluster header nodes by artificial bee colony algorithm on the basis of Protocol LEACH, which takes into account not only energy utilization ratio but also more about nodes energy consumption balance. The iteration results of artificial bee colony algorithm select two cluster-heads for each cluster, namely master and assistant cluster-heads. The master cluster-head collects data inside the cluster and transmit them to assistant cluster-head after information fusion, and assistant cluster-head will transmit these data to base station. Above said algorithm can effectively improve energy utilization ratio, balance network load and extend network lifecycle.

\section{ARTIFICIAL BEE COLONY ALGORITHM}

The artificial bee colony (ABC) algorithm $[5,6]$ imitates bees foraging progress, of which main characteristic is only to make comparison of advantages and disadvantages of the issues and let emergence of global optimum by local optimization behavior of artificial bee individual, whereas no need to know about special information, so as to have rapidly convergence rate.

Artificial bee colony consists of three different categories, namely employed bee, observation bee and scout bee, and each category of bee represents one position, namely one solution. The employed bee accesses previous position and keeps searching for better position around and chooses the better greedily. The observation bee chooses the position shared by employed bee in dancing area with a certain probability and continue to search. The scout bee search for position randomly.

Initialization of artificial bee colony algorithm: The employed bee and observation bee account for half of total bee quantity separately. The position of food source will be generated randomly, each employed 


\section{MATEC Web of Conferences}

bee matches one position, and maximum iterations will be set up.

Iterative process of artificial bee colony algorithm: Each employed bee generates candidate position through equation (1). The advantage and disadvantage of position will be compared by calculation results of position fitness function, and the position will be selected greedily.

$$
v_{i j}=x_{i j}+\theta_{i j}\left(x_{i j}-x_{k j}\right) \text {, }
$$

In equation (1), $\theta_{i j}$ is random number between -1 and $1, v_{i}$ is candidate position, $x_{i}$ is current position, and $x_{k}$ is position of neighbor employed bee $j \in\{1,2, \ldots . . . D\}, D$ is position vector dimension. The employed bee shares position information with observation bee with a certain probability after completing searching. The probability generated via equation (2) by observation bee will select position by means of roulette which guarantees better position will be selected with a higher probability.

$$
p_{i}=\frac{f i t_{i}}{\sum_{n=1}^{S N} f i t_{n}},
$$

In equation (2), fit ${ }_{i}$ represents fitness function of position $i, S N$ represents total number of positions. The observation bee will become employed bee after completing position selection, executing searching process. When the time of a position not being replaced by the relevant candidate reaches up to the limit, this position may be locally optimal solution; when the position is abandoned, the employed bee becomes scout bee, randomly generating a position via equation (3), so as to jump out of locally minimum solution.

$$
x_{i}^{j}=x_{\min }^{j}+\operatorname{rand}(0,1)\left(x_{\max }^{j}-x_{\min }^{j}\right),
$$

In equation (3), $x_{i}$ is abandoned solution $j \in\{1,2, \ldots \ldots . D\}, x_{\min }^{j}$ and $x_{\max }^{j}$ are minimum and maximum that parameter may take under index $j$.

\section{CLUSTERING ROUTING ALGORITHM BASED ON ARTIFICIAL BEE COLONY AND ASSISTANT CLUSTER-HEAD(ABC-A)}

\subsection{Assistant cluster-head selection}

In model of single cluster-head, cluster-head undertakes member data collection, information fusion, and communication with base station, etc. Too heavy energy load causes cluster header nodes to die early, and also shorter time of death and survival of better position's cluster-head selected by artificial bee colony, which will pose impact to overall network performance. Therefore, assistant cluster-head mechanism is introduced in cluster to balance network energy load.
For every node $b_{i}$ inside the cluster, calculating quadratic sum for distance $d\left(M C H, b_{i}\right)$ between node $b_{i}$ and master cluster-head $M C H$, and distance $d(M C H, B S)$ between node $b$ and base station $B S$; selecting the minimum of above quadratic sum as assistant cluster-head $A C H$, therefore, $A C H$ satisfies equation (4).

$d(M C H, A C H)+d(A C H, B S)=$

$\min _{i=1 \ldots . . m_{i}}\left(d\left(M C H, b_{i}\right)+d\left(b_{i}, B S\right)\right)$

\subsection{ABC-A algorithm process}

ABC-A algorithm is based on Protocol LEACH [3], and runs on a round cycle, each round consists of cluster-head selection stage, network stage and stabilization stage. $\mathrm{ABC}-\mathrm{A}$ is centralization clustering routing algorithm, cluster-head selection result is calculated by base station and broadcasted. During cluster-head selection stage, artificial algorithm generates two cluster-heads in each cluster, namely master and assistant cluster-heads; in network stage, non-cluster header node selects nearest master cluster-head to join in the cluster; in stabilization stage, master cluster-head distributes schedule table, TDMA communication method is adopted inside the cluster, assistant cluster-head communicates with base station by CDMA in order to avoid inter-cluster interference.

This article assumes each node can directly communicate with any nodes in the network as well as base station, and also can calculate distance between each other. Meanwhile, base station knows about relative distance of all nodes, the data collected by neighbor node has relatively higher similarity, and the cluster header nodes can fuse cluster member node data into a data package with fixed length.

Assuming there are $p$ nodes and $q$ cluster-heads in the network, detailed ABC-A algorithm as follows:

1) Initial phase: initialization generates $S N$ candidate solution, of which dimension is $q$, each candidate solution randomly selects $q$ different nodes among $p$ nodes, namely master cluster-head $M C H$, iterations number is set to be one.

2) Iterative phase

(1) For each node $p_{i}$ in the network, calculating distance $d\left(p_{i}, M C H_{i}\right)$ between node $p_{i}$ and cluster header nodes of master cluster-head $M C H$, selecting nearest cluster-head $k$ to join, which satisfies equation (5).

$$
d\left(p_{i}, M C H_{k}\right)=\min _{j=1,2, \ldots q}\left(d\left(p_{i}, M C H_{j}\right)\right) .
$$

(2) For each cluster, calculating quadratic sum for distance $d\left(M_{C H}, b_{j}\right)$ between each node $b_{i}$ and master cluster-head $\mathrm{MCH}_{j}$, and distance $d\left(b_{i}, B S\right)$ between $\quad b_{i}$ and base station $B S$. Greedily select cluster member as assistant cluster-head according to minimum quadratic sum standard.

(3) Calculating fitness function of each solution, which determines whether the solution is good or bad. 
Executing artificial bee colony algorithm to update position, recording optimal solution's master cluster-head set and assistant cluster-head set.

(4) Iterative times +1 , judging if it reaches the maximum iterative times. If yes, stop; otherwise, iterative continues.

3) Network phase:

non cluster header nodes join in nearest cluster of master cluster header nodes, after optimal solution selection for master cluster-head set and assistant cluster-head set is completed.

4) Stabilization phase:

TDMA communication method is adopted between cluster member and master cluster-head, master cluster-head and assistant cluster-head, CDMA communication method is adopted between assistant cluster-head and base station.

\subsection{Fitness function}

In $\mathrm{ABC}-\mathrm{A}$ algorithm, one solution's fitness function represents the solution's advantage and disadvantage, therefore, fitness function selection is a critical issue.

Majority of energy consumption of wireless sensor networks comes from communication ${ }^{[7]}$; the energy consumed by communication is much more than by sensing and calculation. This article ignores the energy consumed by calculation and storage. The routing protocol of wireless sensor networks aims at improving energy utilization ratio, and extending network lifetime. On the basis of Protocol ABC ${ }^{[2]}$, in model of single cluster-head, when taking into account overall networks energy consumption, the result of fitness function has inverse relationship with overall networks energy consumption under the situation of cluster-head set corresponding to the solution. Fitness function $f^{\text {energy_consume }}$ can be calculated according to equation (5) (6) (7):

$$
\begin{aligned}
& f^{\text {energy_consume }}=\left[\sum_{i=1}^{c}\left(m_{i} \cdot E^{R X}+E_{i}^{T X}+\sum_{j=1}^{m_{i}} E_{j}^{T X}\right)\right]^{-1}, \\
& E^{T X}=E^{\text {elec }} \cdot k+E^{a m p} \cdot k \cdot d^{2},
\end{aligned}
$$$$
E^{R X}=E^{\text {elec }} \cdot k
$$

In above equations, $c$ represents the number of cluster; $m_{i}$ represents the number of cluster $i$ member. $k$ represents bit number of data package; $d$ represents communication distance; $E^{\text {elec }}$ represents basic power consumption coefficient of transceiver circuit; $E^{a m p}$ represents power consumption coefficient of power amplifier circuits; $E^{R X}$ represents energy consumption of accepting data; $E^{T X}$ represents energy consumption of sending data. In the model of single cluster-head, when taking account into overall networks energy consumption and residual energy of cluster header nodes, the network energy load can be balanced to some extent, relevant fitness

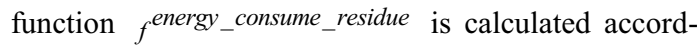
ing to equations (9) and (10):

$$
\begin{aligned}
& f^{\text {energy_consume_residue }}= \\
& \beta \cdot f^{\text {energy_consume }}+(1-\beta) \cdot f^{\text {energy_residue }} \\
& \text { fitness }^{\text {energy_consume }}=\sum_{i=1}^{c} E_{i}^{\text {current }} \text {, }
\end{aligned}
$$

Where, $\beta$ is weight factor; $E_{j}^{\text {current }}$ is residual energy when current cluster header nodes are normalized.

\subsection{Fitness function of ABC-A algorithm}

As introducing assistant cluster-head mechanism, data transmission can be divided into three parts: (1) communication between cluster member and master cluster-head; (2) master cluster-head make fused data communicate with assistant cluster-head; (3) communication between assistant cluster-head and base station. Therefore, in ABC-A algorithm, fitness function $f^{\text {assistant }}$ has changes accordingly, which can be calculated according to equations (7), (8) and (11):

fitness assistant $=\left[\sum_{i=1}^{c}\left(m_{i} \cdot E^{R X}+E_{\text {in }}^{T X}+E_{\text {in }}^{R X}+E_{\text {out }}^{T X}+\sum_{j=1}^{m_{i}} E_{j}^{T X}\right)\right]^{-1}$

Where, $E_{i n}^{T X}$ represents energy consumption of master cluster-head and assistant cluster-head; $\quad E_{\text {in }}^{R X}$ and $E_{\text {out }}^{T X}$ represents energy consumption of assistant cluster-head accepting data, and communication with base station respectively; $E_{i n}^{T X}$ represents energy consumption of data transmission by master cluster-head and assistant cluster-head; $E_{i n}^{R X}$ represents energy consumption of assistant cluster-head accepting data; $E_{\text {out }}^{T X}$ represents communication energy consumption between assistant cluster-head and base station; $\sum_{j=1}^{m_{i}} E_{j}^{T X}$ represents energy consumption of cluster member transmitting data.

\section{SIMULATION EXPERIMENT}

In simulation experiment, 100 sensor nodes are distributed randomly in area $500 \mathrm{~m} \times 500 \mathrm{~m}$, base station position is $(250 \mathrm{~m}, 575 \mathrm{~m})$, initial energy of each node

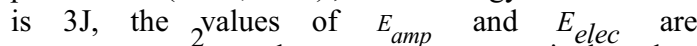
$100 \mathrm{pJ} / \mathrm{bit} / \mathrm{m}^{2}$ and $50 \mathrm{~nJ} / \mathrm{bit}$ respectively, data packet size is 8192 bit , the number of network cluster is five $[8,9]$. The number of current network nodes remaining is less than 20 , network stops working. 


\section{MATEC Web of Conferences}

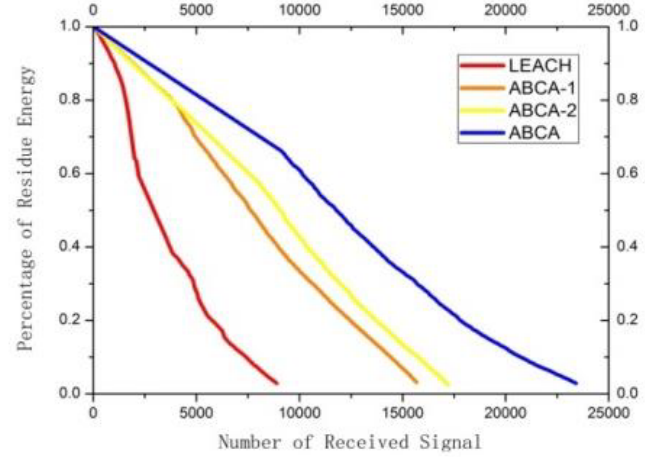

Figure 1. The relation diagram of residual energy percentage and volume of accepting data

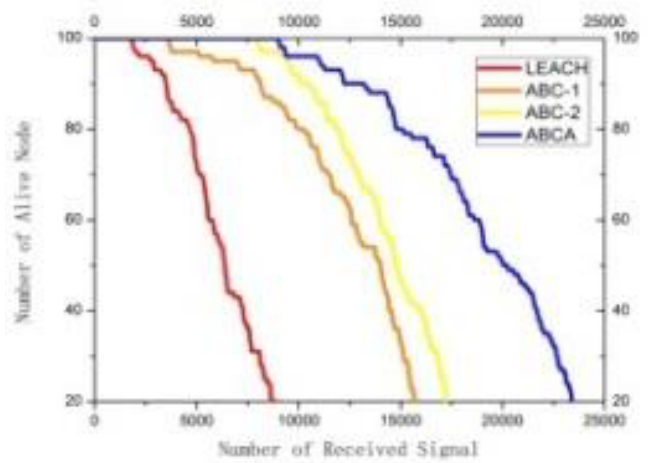

Figure 2. The relation diagram for number of survived nodes and volume of accepting data

Figure 1 is a relation diagram for residual energy percentage and volume of accepting data, $\mathrm{ABC}-1$ is results of fitness function $f^{\text {energy_consume }}, \mathrm{ABC}-2$ is the results of fitness $f^{\text {energy_consume_residue }}$ taking the optimal weighting factor $\beta=0.5, \mathrm{ABC}-\mathrm{A}$ is the result of fitness function $f^{\text {assistant }}$, namely the algorithm introduced in this article. Figure 2, under the same condition, is relation diagram for number of survived nodes and volume of accepting data. Because the artificial bee colony algorithm optimizes cluster-head selection process, selecting the optimal cluster-head, we improve energy utilization ratio. Meanwhile, assistant cluster-head mechanism balances network energy load and extends network lifecycle. The experiment shows that when network has less than 20 survived nodes and stops working, for parameter of accepting data volume, ABC-A algorithm increases by approximately $35 \%$ than $\mathrm{ABC}-2$, increases by approximately $53 \%$ than $\mathrm{ABC}-1$, and increases by approximately $151 \%$ than Protocol LEACH. Protocol ABC is to calculate the distance between each node and cluster-head, Protocol $\mathrm{ABC}-\mathrm{A}$ is to calculate the distance between each node and cluster-head and traverse all nodes for assistant cluster-head, and only constant term changes con- cerning complexity, so they have same time complexity.

\section{CONCLUSION}

Extending network lifecycle is one of major goals for wireless sensor networks, so the solution presented in this article is, based on introducing assistant cluster-head, a clustering routing algorithm that uses the artificial bee colony algorithm to select master and assistant cluster-heads. In terms of performance index such as volume of accepting data, when network stops working, this algorithm increases by approximately $35 \%$ than ABC-2 algorithm taking into account network energy consumption and residual energy of cluster header nodes, increases by approximately $53 \%$ than ABC-1 algorithm only taking into account network energy consumption, and increased by approximately $151 \%$ than LEACH algorithm. The experiment shows that $\mathrm{ABC}-\mathrm{A}$ algorithm effectively improves energy utilization ratio, balances network energy load, and extends network lifecycle.

\section{REFERENCES}

[1] I. Akyildiz, W. Su, Y. Sankarasubramniam \& E. Cayirci. 2002. Wireless sensor networks: a survey, Comput. Netw., 8: 393-422.

[2] J. Yick, B. Mukherjee \& D. Ghosal. 2008. Wireless sensor network survey. Comput netw, 52: 2292-2330.

[3] W. Heinzelman, A. Chandrakasan. \& H. Balakrishnan. 2002, Energy-efficient communication protocol for wireless microsensor networks, Proc. Hawaaian Int. Conf. on Systems Science.

[4] D. Karaboga, S. Okdem \& C. Ozturk. 2012. Cluster based wireless sensor networks routing using artificial bee colony algorithm. Wireless Netw., 18: 847-860.

[5] D. Karaboga. 2005. An idea based on honey bee swarm for numerical optimization, Technical report-TR06, Erciyes University, Engineering Faculty, Computer Engineering Department.

[6] D. Karaboga, B. Gorkemil, C. Ozturk \& N. Karaboga. 2012. A comprehensive survey: artificial Intelligence Review, Artif. Intell. Rew., 42: 21-57.

[7] G. Anastasi, M. Conti, A. Falchi \& E. Gregori. 2004. A Passarella, Performance measure of motes sensor networks, ACM Press, pp.174-181.

[8] Y. Liang \& H. Yu. 2005. PSO-based energy efficient gathering in sensor networks, LNCS, 3794: 362-369.

[9] N.M. A. Latiff \& B.S. Sharif. 2007. Performance comparison of optimization algorithms for clustering in wireless sensor networks, Int. Conf. on Mobile adhoc and sensor systems, pp.1-4. 